\title{
Inhibition-Induced Forgetting Results from Resource Competition between Response Inhibition and Memory Encoding Processes
}

\author{
이 Yu-Chin Chiu (丘雨勤) and Tobias Egner \\ Center for Cognitive Neuroscience, Duke University, Durham, North Carolina 27708
}

Response inhibition is a key component of executive control, but its relation to other cognitive processes is not well understood. We recently documented the "inhibition-induced forgetting effect": no-go cues are remembered more poorly than go cues. We attributed this effect to central-resource competition, whereby response inhibition saps attention away from memory encoding. However, this proposal is difficult to test with behavioral means alone. We therefore used fMRI in humans to test two neural predictions of the "common resource hypothesis": (1) brain regions associated with response inhibition should exhibit greater resource demands during encoding of subsequently forgotten than remembered no-go cues; and (2) this higher inhibitory resource demand should lead to memory encoding regions having less resources available during encoding of subsequently forgotten no-go cues. Participants categorized face stimuli by gender in a go/no-go task and, following a delay, performed a surprise recognition memory test for those faces. Replicating previous findings, memory was worse for no-go than for go stimuli. Crucially, forgetting of no-go cues was predicted by high inhibitory resource demand, as quantified by the trial-by-trial ratio of activity in neural "no-go" versus "go" networks. Moreover, this index of inhibitory demand exhibited an inverse trial-by-trial relationship with activity in brain regions responsible for the encoding of no-go cues into memory, notably the ventrolateral prefrontal cortex. This seesaw pattern between the neural resource demand of response inhibition and activity related to memory encoding directly supports the hypothesis that response inhibition temporarily saps attentional resources away from stimulus processing.

Key words: attention; cognitive control; memory; response inhibition

\section{Significance Statement}

Recent behavioral experiments showed that inhibiting a motor response to a stimulus (a "no-go cue") impairs subsequent memory for that cue. Here, we used fMRI to test whether this "inhibition-induced forgetting effect" is caused by competition for neural resources between the processes of response inhibition and memory encoding. We found that trial-by-trial variations in neural inhibitory resource demand predicted subsequent forgetting of no-go cues and that higher inhibitory demand was furthermore associated with lower concurrent activation in brain regions responsible for successful memory encoding of no-go cues. Thus, motor inhibition and stimulus encoding appear to compete with each other: when more resources have to be devoted to inhibiting action, less are available for encoding sensory stimuli.

\section{Introduction}

Response inhibition, the ability to preempt or cancel goalinappropriate actions, is considered a core cognitive control

Received Feb. 6, 2015; revised July 22, 2015; accepted July 24, 2015.

Author contributions: Y.-C.C. and T.E. designed research; Y.-C.C. performed research; Y.-C.C. analyzed data; Y.-C.C. and T.E. wrote the paper.

This work was supported in part by National Institute of Mental Health Award R01 MH 087610 to T.E. The authors declare no competing financial interests.

Correspondence should be addressed to Dr. Yu-Chin Chiu, Center for Cognitive Neuroscience, Duke University, LSRC, Box 90999, Durham, NC 27708. E-mail: chiu.yuchin@duke.edu.

DOI:10.1523/JNEUROSCI.0519-15.2015

Copyright $\odot 2015$ the authors $\quad 0270-6474 / 15 / 3511936-10 \$ 15.00 / 0$ function (Logan and Cowan, 1984; Aron, 2007), an impairment that contributes to impulsive symptoms of multiple psychiatric diseases, including obsessive-compulsive disorder, substance abuse, and attention-deficit/hyperactivity disorder (Horn et al., 2003; de Wit, 2009). However, the relation of response inhibition to other cognitive control functions, and to traditional cognitive domains, such as perception, memory, and attention, remains poorly understood (Jurado and Rosselli, 2007; Miyake and Friedman, 2012).

A recent behavioral study has shed new light on this issue by documenting an "inhibition-induced forgetting" effect, whereby inhibiting responses to no-go or stop cues impaired subsequent 
memory for those cues, compared with go cues (Chiu and Egner, 2015). Moreover, participants were less likely to detect occasional perceptual probes displayed on no-go cues than on go cues (Chiu and Egner, 2015) (Experiment 5). Together, these data foster the novel hypothesis that response inhibition competes for a common (attentional) resource with memory encoding processes, thus leading to poorer memory for no-go cues (Chiu and Egner, 2015). However, this "common resource hypothesis" is difficult to validate with behavioral data alone.

The inhibition-induced forgetting effect is reliable but subtle, as only a subset of no-go stimuli are forgotten. Our question, therefore, is as follows: what is special about these forgotten no-go trials? The common resource hypothesis suggests that these trials should be characterized by particularly strong resource demand by the inhibitory control process, which saps attention away from mnemonic stimulus encoding. This translates into the neural predictions that brain regions associated with response inhibition, relative to those involved in response execution, should exhibit greater activation (reflecting greater resource demands) during encoding of subsequently forgotten no-go cues than remembered ones; moreover, these no-go trials of exceptionally high resource-demand should consequently be associated with less activation (indicative of sapped resources) in brain regions associated with memory encoding success.

To examine these predictions, we here combined fMRI with a go/no-go task, followed by a surprise recognition memory test for go/no-go cues. Based on the logic of the "interactive race model" of response inhibition (Boucher et al., 2007), we developed an index that quantified trial-by-trial inhibitory neural resourcedemand by gauging the trial-based ratio of activation in the brain networks responsible for response inhibition ("no-go network" activity) versus response execution ("go network" activity) (see Materials and Methods). We predicted that this ratio of no-go/go network activation, reflective of inhibitory resource demand, would be higher for no-go cues that were later forgotten than those that were later remembered. We then tested whether this trial-by-trial index of inhibitory resource demand exhibits a negative correlation with activity in brain regions associated with encoding success, indicative of response inhibition sapping resources away from mnemonic encoding.

To preview the results, our imaging data confirmed both of the above predictions of the common resource hypothesis. By revealing the neural mechanisms underlying the inhibitioninduced forgetting effect, these data enhance our understanding of the cognitive architecture of executive function.

\section{Materials and Methods}

\section{Participants}

Twenty-four right-handed volunteers (mean \pm SD age, $24.4 \pm 4.3$ years; 14 men, 10 women) gave written informed consent to participate in this study, which was approved by the Duke University Health System Institutional Review Board. All participants had normal or corrected-tonormal vision and reported no history of neurological or psychiatric disorders. Participants were compensated with $\$ 30$ for their time (1.5 h). Data of 3 participants were excluded: two due to excessive movement during the scan and one due to loss of behavioral data (malfunction of the response box).

\section{Stimuli}

A total of 240 unique identity grayscale photographs of affectively neutral faces (half males/females) were used in the main experiment. These images were drawn from the Productive Aging Laboratory Face Database (Minear and Park, 2004), the Cohn-Kanade Facial Expression Data Base (Kanade et al., 2000), the Georgia Tech Face Database (http://www. anefian.com/research/face_reco.htm), and a collection by Endl et al.
(1998). Half of the face images appeared initially in the go/no-go task and subsequently served as the "old" stimuli in the recognition memory task; the other half only appeared in the recognition memory task where they served as the "new" stimuli. The face stimulus assignment to old/new conditions was randomized across participants. The filler task (encoding-retrieval delay) used 120 grayscale photographic house images collected from real estate websites. Half of these images were of one story houses and the other half were of two story houses. All stimuli subtended $\sim 4^{\circ}$ in height and $3^{\circ}$ in width.

\section{Procedure}

Before entering the scanner, participants completed a practice block of 20 trials to familiarize themselves with the assigned stimulus-response mapping. Half of the participants were instructed to respond to male faces by pressing a button with their right index finger (i.e., go trials) but not to press any key in response to female faces (i.e., no-go trials). The other half of the participants received the opposite gender-to-go/no-go mapping. In the scanner, participants first performed 4 runs of the face-gender go/no-go task, with each run including one repetition per face (Fig. 1a). We chose four repetitions because this number should be sufficient to induce stimulus-response and stimulus-inhibition associations (Verbruggen and Logan, 2008), and to avoid a floor effect in the subsequent recognition memory task based on our previous study (Chiu and Egner, 2015).

Each trial started with a fixation cross at the center of the screen for 250 $\mathrm{ms}$, followed by a face stimulus for $800 \mathrm{~ms}$, during which participants could make a response. The response deadline was the same as the duration of the face stimulus (i.e., $800 \mathrm{~ms}$ ). If a response was not made within the response deadline, this trial was counted as a no-go trial. Participants then received a $1 \mathrm{~s}$ on-screen feedback ("correct" or "incorrect"). Participants were instructed to respond as fast as they could while being accurate. Responses made later than the response deadline were considered incorrect trials. Intertrial intervals were randomly drawn from a pseudoexponential distribution of $2.5-4.5 \mathrm{~s}$ ( step size $=250 \mathrm{~ms}$ ). After 4 runs of the go/no-go task, participants performed a "filler task," which served as the encoding-retrieval interval (5 $\mathrm{min})$. In that task, participants were presented with house stimuli and were instructed to categorize the images according to whether they showed one or two story houses. Trial timing in the filler task was the same as the go/no-go task, except that the intertrial intervals were fixed to $2 \mathrm{~s}$.

After the filler task, participants performed 2 runs of a surprise memory recognition task that tested their memory for the face images encountered in the go/no-go task. The recognition memory task included all of the "old" faces from the go/no-go task (120 identities), intermixed with an equal number of new faces (120 identities). On each trial, a stimulus was presented for $2 \mathrm{~s}$ during which participants selected one of the following responses: "definitely new," "probably new," "probably old," or "definitely old" (mapped onto the four buttons held by the participant from left to right or right to left, respectively). Only responses made during the $2 \mathrm{~s}$ window were considered. Intertrial intervals were randomly drawn from a pseudo-exponential distribution of 2.5-4.5 s (step size $=250 \mathrm{~ms}$ ).

\section{fMRI acquisition}

Images were acquired on a 3.0-T GE MR750 scanner using an 8-channel head coil. Functional images were acquired parallel to the AC-PC plane with a T2*-weighted single-shot gradient EPI sequence of 40 contiguous axial slices $\left(\mathrm{TR}=2000 \mathrm{~ms}\right.$; TE $=28 \mathrm{~ms}$, flip angle $\left.=90^{\circ}\right)$. The field of view was $192 \mathrm{~mm} \times 192 \mathrm{~mm}$, with a $64 \times 64$ matrix, yielding an in-plane resolution of $3 \mathrm{~mm} \times 3 \mathrm{~mm}$. Structural images were acquired with a T1-wighted SPGR axial sequence $(\mathrm{TR}=7.912 \mathrm{~ms}, \mathrm{TE}=3.152 \mathrm{~ms}$, field of view $=256 \mathrm{~mm} \times 256 \mathrm{~mm}$ ) parallel to AC-PC plane using a 120 slices of $1 \mathrm{~mm}$ thickness and an in-plane resolution of $1 \mathrm{~mm} \times 1 \mathrm{~mm}$. The go/no-go task was acquired in 4 runs of 184 images each, and the memory task was acquired in 2 runs of 186 images each. However, images collected during the retrieval phase were not further analyzed as we were primarily interested in neural activity during the encoding phase (i.e., go/no-go task). 


\section{fMRI preprocessing}

All preprocessing and statistical analysis was performed using SPM8 (http://www.fil.ion. ucl.ac.uk/spm/software/spm8/). The first four images of each functional run were discarded as these were acquired to allow for saturation of the MR signal before the onset of the task. Functional data were slice-time corrected and realigned to the mean image of all functional volumes. Each subject's EPI volumes were coregistered to that subject's anatomical scan. The anatomical scan was then normalized to the MNI template brain, and the resulting transformation parameters were applied to the EPI volumes to achieve spatial normalization. Normalized functional data were resampled into $3 \times 3 \times 3 \mathrm{~mm}$ voxel size and smoothed using an $8 \mathrm{~mm}^{3}$ Gaussian kernel.

\section{fMRI analyses}

Definition of go and no-go networks. The standard GLM approach (Friston et al., 1994) was used to regress the functional brain data against task models and to estimate $\beta$ parameters for conditions of interest. To identify brain regions associated with response inhibition and response execution, the go/no-go task was modeled with 4 regressors of interest coding for onsets of correct no-go, correct go, incorrect no-go, and incorrect go trials. The trials were modeled as mini boxcar functions of $1.8 \mathrm{~s}$ duration, capturing both the stimulus duration and the subsequent feedback, and convolved with a canonical hemodynamic response function. In addition, realignment parameters were modeled as nuisance regressors to account for motion artifact. Two within-subject linear contrasts (i.e., correct go $>$ correct no-go and correct no-go $>$ correct go) were performed, and the resulting contrast images were entered into group analyses that treated subjects as random effects. The group-level results were corrected for multiple comparisons to yield a whole-brain $\alpha<0.01$ with an initial voxelwise threshold of $t=$ $2(p<0.03)$ in combination with a cluster size threshold of 60 . This number was determined by running 3dClustSim (http://afni.nimh.nih. gov/pub/dist/doc/program_help/3dClustSim.html) with the estimated intrinsic smoothness based on residual images in the analysis. The go network was defined by significant voxel clusters in the go > no-go map, and the no-go network by significant voxel clusters in the no-go $>$ go map.

Network activity ratio analysis. To test the hypothesis that trial-by-trial variation in the resource demands of response inhibition mediated the later forgetting of a subset of no-go cues, we performed a novel activity ratio analysis. This analysis computed the ratio of no-go to go network activation on a trial-by-trial basis (see above Definition of go and no-go networks). We used activity in the go network as the denominator to no-go network activity for two reasons. First, this ratio approach serves to normalize no-go network activation across subjects. As the ratio is calculated within each subject, it circumvents possible problems with comparing absolute activation levels in the two networks across subjects. Also, this activity ratio metric expresses a relative demand on inhibitory resources (the larger the ratio, the greater the demand) across trials, but due to the possibility of different activity scaling or baseline activation levels within each network, we refrain from attributing significance to the absolute value of that ratio. In other words, we do not suppose that the no-go/go network activity ratio would have to be greater than one in order for a response to be inhibited; what matters are relative differences in the ratio across trials. Second, the relative activation levels between these networks should correspond closely to relative resource demands by the inhibitory network within the framework of the interactive race model of response inhibition (Boucher et al., 2007), an extension of the standard race model that has been shown to provide a more realistic account of the neural dynamics underlying response inhibition. In this model, response inhibition is conceived of alternate motor and inhibitory processes that race for the earliest finish time (Logan and Cowan, 1984), and the two processes are interactive in that they are mutually inhibitory (Boucher et al., 2007). Taking activity in the go network as the denominator of our ratio analysis accounts for this proposed interaction between the go and no-go processes. Specifically, a trial with a higher no-go/go network activity ratio indicates that greater neural activation is required for the inhibitory process to outrace the go process compared with a trial with a lower ratio. Moreover, the common resource hypothesis assumes that no-go trials that incur greater resource demands, that is, no-go trials with a high no-go/go activity ratio, leave less resources available for the concurrent process of encoding the no-go cue into memory.

The ratio analysis involved four steps. First, we obtained a single-trial $\beta$ estimate of activation using a least-square-separate method (Mumford et al., 2012). In brief, this method entailed running a separate GLM for each trial where the trial is modeled as the regressor of interest, and all other trials are combined into a single nuisance regressor. Second, we then calculated, for each trial, the mean $\beta$ estimate of all voxels in two masks: one consisting of voxels in the go network and the other consisting of voxels in the no-go network. Third, we derived the activity ratio for each trial by dividing the mean $\beta$ in the no-go network by the mean $\beta$ in the go network. Finally, trials were sorted into four conditions by subsequent memory performance: no-go forgotten, no-go remembered, go forgotten, and go remembered. A paired-samples $t$ test was used to test the a priori hypothesis that the mean no-go/go network activity ratio would be larger for forgotten than remembered no-go cues. Although we did not have an a priori hypothesis regarding the relationship of nogo/go network activity ratio and subsequent memory for go trials, we nonetheless conducted an exploratory 2 (action cue: go vs no-go) $\times 2$ (subsequent memory: forgotten vs remembered) ANOVA on the mean activity ratio. These analyses were confined to cues from trials with correct go/no-go decisions in the go/no-go task. 
In addition to this categorical test of a higher no-go/go network activity ratio for forgotten compared with remembered no-go trials, we also sought to connect this inhibitory demand index to subsequent memory in a more continuous fashion. Therefore, we tested whether the trial-bytrial activity ratio predicted graded memory strength as defined by recognition confidence (i.e., "definitely old," "probably old," "probably new," and "definitely new"). To this end, for each participant, these no-go cue memory ratings were converted into numerical scores of 1 , $0.667,0.333$, and 0 , respectively, and a robust regression was performed to estimate the relationship between trialwise no-go/go network activity ratio and subsequent memory strength. Subsequently, a $t$ test was performed on the standardized coefficient estimate at the group level to assess statistical significance.

Definition of memory encoding regions. To test the hypothesis that inhibitory resource demand (as defined in Network activity ratio analysis) diverts resources from memory encoding, we aimed to first define brain regions that are involved in the successful encoding of no-go cues, to then probe whether activity in these regions was relatively suppressed during trials where inhibitory resource demand was high. To identify brain regions that contribute to the encoding of no-go cues, we performed a "difference in memory" (DM) analysis (for review, see Paller and Wagner, 2002). Specifically, we ran a second GLM with go/no-go trials recoded by each subject's subsequent memory performance. The GLM included 5 regressors: no-go remembered, no-go forgotten, go remembered, go forgotten, and miscellaneous trials of no interest (including cues that had no response in the recognition memory task, and error trials in the go/no-go task). Linear contrasts were performed withinsubjects, and the resulting contrast images were entered into a randomeffects group analyses. Group-level maps were corrected for multiple comparisons to yield a whole-brain $\alpha<0.01$ by using an initial voxelwise threshold of $t=2(p<0.03)$ in combination of a cluster size threshold of 57 voxels (determined by $3 \mathrm{dCl}$ (ustSim). The DM effect of primary interest to assessing our hypothesis was the "subsequent remembering effect" for no-go cues, based on the contrast of remembered $>$ forgotten no-go cues. (For completeness sake, we also assessed the "subsequent forgetting effect," based on the contrast of forgotten $>$ remembered no-go cues.)

Ratio correlation analysis. According to our hypothesis, forgotten no-go trials should be characterized by exceptionally high inhibitory resource-demand, and they should consequently be associated with less activation (indicative of sapped resources) in brain regions associated with memory encoding success. Given our hypotheses, our specific focus was on regions normally responsible for successful encoding of no-go cues. We therefore identified regions that selectively predicted successful encoding of no-go cues (see Definition of memory encoding regions) to then interrogate the trial-by-trial activity patterns in these regions with respect to trial-by-trial inhibitory resource demands. We next tested whether voxels within the above-identified regions involved in the successful encoding of no-go cues exhibited (negatively) correlated fluctuations in trial-to-trial activity with our index of resource-demand (i.e., the no-go/go network activity ratio). To this end, we used an analysis approach similar to a $\beta$ series correlation (Rissman et al., 2004). Specifically, for each subject, we first concatenated the single-trial no-go/go activity ratio (see Network activity ratio analysis) of all no-go forgotten trials, forming a ratio series. Similarly, the single-trial $\beta$ estimates of all no-go forgotten trials in a single voxel were also concatenated, forming a $\beta$ series. We then calculated Pearson's correlation coefficients between the ratio and the $\beta$ series and repeated this calculation for each voxel in the mask created by the no-go remembered $>$ no-go forgotten contrast. These correlation coefficients were then transformed into $t$ values. Each subject's $t$ map was then entered into a group-level one-sample $t$ test to access the mapwise significance of the correlation. The group-level map was corrected for multiple comparisons (adjusted for number of voxels in the mask) to yield a whole-brain $\alpha<0.01$ by using an initial voxelwise threshold of $t=2(p<0.03)$ in combination of a cluster size threshold of 30 voxels (determined by $3 \mathrm{dClustSim}$ ).

Anatomical ROI analysis. We also extracted mean activity ( $\beta$ estimates) from the subthalamic nucleus (STN) to test an alternative, "global inhibition account" of the forgetting effect. According to the global inhibition hypothesis, forgetting of no-go cues could arise from a wide-
Table 1. Response times and error rates in the go/no-go task ${ }^{a}$

\begin{tabular}{lllll}
\hline & Repetition 1 & Repetition 2 & Repetition 3 & Repetition 4 \\
\hline $\begin{array}{l}\text { RT for go } \\
\text { trials (ms) }\end{array}$ & $514.23(10.29)$ & $496.21(10.93)$ & $485.51(8.81)$ & $477.44(6.90)$ \\
$\begin{array}{l}\text { Error rate for } \\
\text { no-go trials (\%) }\end{array}$ & $4.60(0.69)$ & $4.21(0.62)$ & $4.37(0.69)$ & $4.21(0.61)$ \\
\hline
\end{tabular}

${ }^{a}$ Data are mean (SEM).

spread inhibition signal sent by the STN that acts not only on the classic motor circuit (Mink, 1996) but also on perceptual processing and memory encoding (Eagle and Baunez, 2010). This global inhibition hypothesis therefore predicts an interaction between action cue (go, no-go) and subsequent memory (remembered, forgotten) in STN activation, where STN activity should be particularly pronounced for forgotten no-go cues. To gauge STN activation, anatomically defined left and right STN ROI masks based on the WFU PickAtlas (Maldjian et al., 2003) were applied to the normalized images of our sample. We included three levels of ROI mask dilation (dilation factor $=1,2,3$ ) to account for possible imprecision in the mapping of subcortical structures between MNI template and individual subject's anatomical images. The mean $\beta$ estimates were extracted and analyzed with a 2 hemisphere (left, right) $\times 2$ action cue (go, no-go) $\times 2$ subsequent memory (forgotten, remembered) ANOVA for each level of dilation to evaluate the global inhibition hypothesis. We included hemisphere as a factor here as prior studies have found asymmetrical involvement of STN in inhibitory control (Aron et al., 2007; Mansfield et al., 2011).

\section{Results}

Behavioral data: inferior recognition memory for no-go than go cues

Participants categorized face stimuli by gender in a go/no-go task and, after a short delay, performed a surprise memory task for those faces (see Materials and Methods). In the go/no-go task, accuracy was similar between go cues (mean \pm SEM, 93.1 \pm 1.25 ) and no-go cues (mean \pm SEM, $95.5 \pm 0.52, t_{(20)}=1.35, p>$ $0.05)$. Go/no-go performance improved with repeated exposure to the go/no-go cues as we found a monotonic decrease in response times (RTs) with increasing repetition (i.e., a significant main effect of repetition on go RTs: $F_{(3,60)}=14.39, p<0.001, \eta_{p}^{2}$ $=0.42$ ) (Fig. $1 b$; Tables 1). However, no-go commission error rates remained at the same level across the four repetitions (main effect of repetition on no-go error rates: $F_{(3,60)}=0.25, p>0.05$, $\eta_{p}^{2}=0.01$ ) (Fig. 1b; Tables 1). Overall, these results indicate that participants performed the go/no-go task as instructed.

Turning to our main goal, we examined the consequences of response inhibition on incidental memory of task stimuli. Only cues from correctly performed go/no-go trials (across all four repetitions) were considered for the subsequent memory analysis. For simplicity, we calculated hit and false alarm rates by collapsing "probably old" and "definitely old" responses and "probably new" and "definitely new" responses in our analyses, distinguishing only between "old" and "new" ratings. We then derived $d^{\prime}$ (i.e., $z$ (hit rate) $-z$ (false-alarm rate) ) as an unbiased index of recognition sensitivity for go and no-go cues, respectively. As expected, the overall recognition hit rate was poor (mean \pm SEM, 68.2 \pm 2.51 ). However, recognition memory was well above chance, as the hit rates for go cues (mean \pm SEM, $71.5 \pm 2.57)$ and no-go cues (mean \pm SEM, $65.0 \pm 3.55)$ were both greater than the false alarm rate (mean \pm SEM, $27.9 \pm 2.76$ ) $(p<.001$; Table 2). Based on our previous experiments (Chiu and Egner, 2015), we examined the directional hypothesis of lower $d^{\prime}$ for no-go cues than for go cues using a one-tailed $t$ test. As expected, $d^{\prime}$ was significantly lower for no-go cues (mean \pm SEM, $1.08 \pm 0.10$ ) than for go cues (mean \pm SEM, $1.26 \pm 0.09$; 
Table 2. Hit and false alarm rates in the recognition memory task ${ }^{a}$

\begin{tabular}{lll}
\hline & \multicolumn{1}{l}{ Cue } & \\
\cline { 2 - 3 } & No-go & Go \\
\hline Hit rate (\%) & $65.00(3.55)$ & $71.55(2.56)$ \\
False alarm rate (\%) & $26.93(3.23)$ & $28.83(2.80)$ \\
\hline
\end{tabular}

${ }^{a}$ Data are mean (SEM).

$t_{(20)}=2.06, p=0.05$, one-tailed, Cohen's $d=0.38$ ) (Fig. $1 c$ ). This result, replicating Chiu and Egner (2015), suggests that response inhibition caused forgetting of a subset of no-go cues. We next addressed the question whether these forgotten no-go trials are characterized by high inhibitory neural resource demands during the go/no-go task.

\section{fMRI data: definition of neural go and no-go networks}

We began by examining brain networks associated with response inhibition and response execution, as identified by contrasting parameter estimates of correct-trial go and no-go cues. As can be seen in Figure $2 a$, no-go compared with go cues elicited greater activation in several frontal regions, including the right and the left inferior frontal gyri, supplementary motor area, right insula, and small clusters in the mid anterior cingulate cortex (Table 3). These regions have been commonly identified in neuroimaging studies of stop-signal and go/no-go tasks (Garavan et al., 1999; Konishi et al., 1999; Liddle et al., 2001; Rubia et al., 2003; Chikazoe, 2010) and are thought to mediate response inhibition. As expected, the reverse contrast of go versus no-go cues showed an increase in activity in a network of brain regions that control movement, including primary motor area, the basal ganglia and cerebellum (Fig. $2 b$; Table 3 ).

Our go/no-go task is intentionally designed to have an equal number of go and no-go trials, to preclude a confounding effect of biased stimulus frequency on subsequent memory. Given that many previous studies of response inhibition have used a greater frequency of go than no-go trials in their design (Garavan et al., 1999; Konishi et al., 1999) to impose greater inhibitory demands on no-go trials, one may wonder whether the current go/no-go task represents a comparable probe of inhibitory control, or whether it may instead resemble more closely standard 2-alternative forced choice tasks that place demands on response selection rather than on response inhibition. One argument in favor of our task gauging response inhibition on no-go trials stems from the fMRI findings themselves. First, if go and no-go trials both primarily involved response selection demands, then the contrast between the two trial types should cancel out response selection related activity. Instead, we observed higher activation in no-go compared with go trials in a set of brain regions that seems to closely parallel findings in prior (frequency-biased) go/no-go and stop-signal studies (Garavan et al., 1999; Konishi et al., 1999; Rubia et al., 2003; Chikazoe, 2010).

However, to provide a quantitative evaluation of whether the no-go network activation we observed (Fig. $2 a$ ) is more likely to reflect the neural signature of response inhibition than that of response selection, we compared our empirical data with meta-analytic maps generated by the term-based meta-analyses tool in NeuroSynth (http://neurosynth.org/) (Yarkoni et al., 2011). To this end, we first generated a forward inference map [i.e., $\mathrm{P}($ activation $\mid$ term)] based on the term "response inhibition" and another one based on "response selection," and we subsequently measured the number of positive voxels in our nogo $>$ go map that were also present in each of the meta-analytic maps. We found that our no-go $>$ go contrast map overlaps to a greater extent with the response inhibition meta-analytic map (by 1027 voxels) than with the response selection map. To estimate the probability of obtaining this difference score of 1027 voxels by chance alone, we generated a null distribution of difference scores by bootstrapping our no-go $>$ go data. Specifically, we shuffled the location of no-go $>$ go positive voxels and then measured the number of voxels in each meta-analytic map to calculate the difference score, using 1000 iterations. This analysis yielded a probability of $p=0.039$ for obtaining a difference score of 1027 (or more), suggesting that our no-go > go contrast map is more consistent with activation patterns associated with "response inhibition" than those associated with "response selection" documented in previous neuroimaging studies. Moreover, it should also be noted that we have shown previously that, when participants were asked to treat the face gender categorization task as a 2-alternative forced choice task (pushing one button for male faces and another button for female faces) instead of a go/ no-go task, no differential effects on subsequent memory occurred (Chiu and Egner, 2015) (Experiment 4).

As an additional test of whether the present, frequencybalanced go/no-go task is comparable with more traditional, frequency-biased versions of this task, we also examined the socalled "inhibitory load effect," which refers to the finding that error rates and no-go network activity on no-go trials scale positively with the number of preceding go trials (Durston et al., 2002; Thomas et al., 2009). Within our dataset, it was feasible to analyze trial history effects up to N-3 (i.e., we could compare behavior and neural activation for no-go trials as a function of whether they were preceded by 1, 2, or 3 go trials). First, as expected, no-go commission error rate increased linearly with the number of preceding go trials (one-way ANOVA with 1, 2, or 3 preceding go's, $F_{(2,40)}=5.54, p<0.01$; linear trend, $F_{(1,20)}=$ $9.68, p<0.01$ ) (Fig. $2 c$, top). Second, the neural data followed the same pattern, with the mean activity in the no-go network increasing linearly with the number of preceding go trials (one-way ANOVA $F_{(2,40)}=3.17, p=0.05$; linear trend, $F_{(1,20)}=5.37, p<$ 0.05 ) (Fig. $2 c$, bottom). These results replicate previous go/no-go studies, documenting that as "inhibitory load" increased (Durston et al., 2002; Thomas et al., 2009), participants indeed committed more no-go errors, and this was accompanied by increasing engagement of the no-go network. Collectively, these meta-analytic and trial history results strongly suggest that, despite the fact that the present go/no-go task entailed an equal number of go and no-go trials, it nevertheless placed inhibitory demands on no-go trials in a manner that seems comparable with previous studies that used unbalanced trial frequencies.

\section{Greater inhibitory resource demands during memory encoding of subsequently forgotten no-go cues}

Next, we asked whether forgetting of no-go cues was associated with greater trial-specific inhibitory resource demands as reflected in the no-go/go network activity ratio (see Materials and Methods). In line with our a priori hypothesis, a paired $t$ test revealed that the no-go/go network activity ratio was significantly greater for no-go cues that were later forgotten than for those that were later remembered (no-go forgotten: mean $=0.68$, no-go remembered: mean $=0.53, t_{(20)}=2.33, p<0.05$, Cohen's $d=$ 0.52 ) (Fig. 3a). Furthermore, to test whether the no-go/go network activity ratio also predicted memory strength in a more continuous fashion, we performed a regression analysis to estimate the relationship between the network activity ratio and graded memory ratings (i.e., definitely old $=1$, probably old $=$ 
a No-go network (No-go > Go)

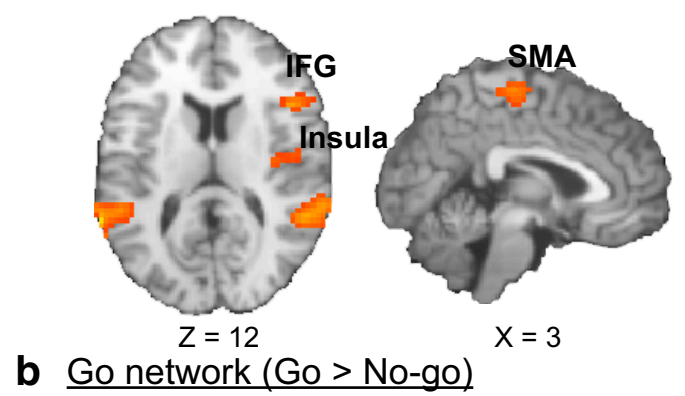

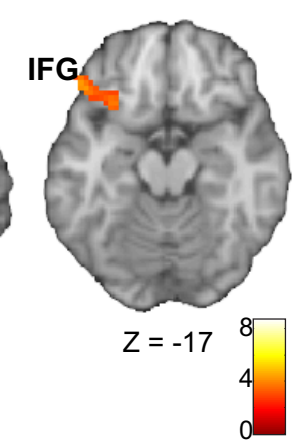

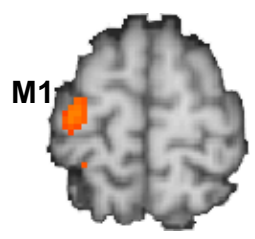

$Z=67$

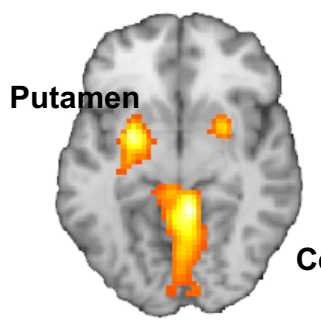

$Z=-8$

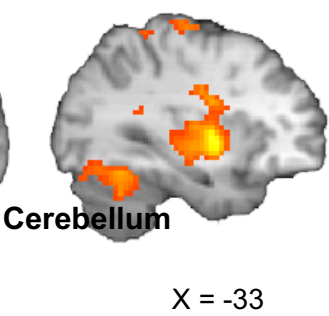

C
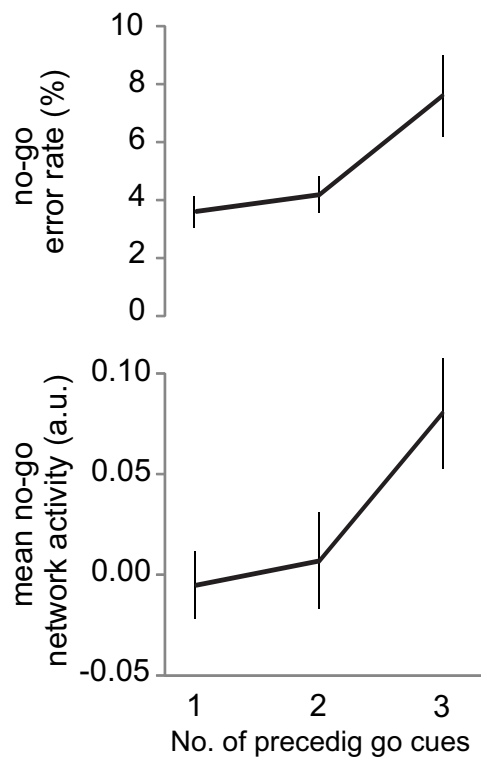

Figure 2. Go and no-go networks and the inhibitory load effect. $\boldsymbol{a}$, The no-go network, associated with response inhibition, was identified via the no-go $>$ go contrast (whole-brain corrected to $\alpha<0.01$ ). $\boldsymbol{b}$, The reverse contrast identified the go network, associated with response execution (whole-brain corrected to $\alpha<0.01$ ). c, As "inhibitory load" increased (defined by an increasing number of preceding go cues), number of commission errors to no-go cues (top) and mean no-go network activity (bottom) also linearly increased. Error bars indicate SEM. Image display according to neurological convention (left is left).

Table 3. Activation clusters for no-go versus go cues in the go/no-go task (wholebrain corrected to $\alpha<0.01$ )

\begin{tabular}{|c|c|c|c|c|c|c|}
\hline \multicolumn{3}{|c|}{ MNI coordinates } & \multirow[b]{2}{*}{ Peak $t$} & \multirow{2}{*}{$\begin{array}{l}\text { No. of } \\
\text { voxels }\end{array}$} & \multirow[b]{2}{*}{ Hemisphere } & \multirow[b]{2}{*}{ Region } \\
\hline$x$ & $y$ & $z$ & & & & \\
\hline \multicolumn{7}{|c|}{ No-go $>$ go } \\
\hline 12 & 11 & 64 & 4.53 & 237 & $\mathrm{R}$ & Supplementary motor area \\
\hline 12 & -10 & 46 & 3.49 & 67 & $\mathrm{R}$ & Cingulate gyrus \\
\hline 54 & -1 & 43 & 8.69 & 722 & $\mathrm{R}$ & Precentral gyrus \\
\hline 48 & 20 & 13 & 4.31 & 258 & $\mathrm{R}$ & Inferior frontal gyrus \\
\hline 57 & -40 & -2 & 5.30 & 651 & $\mathrm{R}$ & Superior temporal gyrus \\
\hline 39 & 2 & -47 & 3.57 & 122 & $\mathrm{R}$ & Inferior temporal gyrus \\
\hline-60 & -25 & -5 & 5.29 & 400 & $\mathrm{~L}$ & Middle temporal gyrus \\
\hline-51 & 29 & -14 & 5.06 & 474 & $\mathrm{~L}$ & Inferior frontal gyrus \\
\hline \multicolumn{7}{|c|}{$\mathrm{G}_{0}>\mathrm{no}-\mathrm{g} 0$} \\
\hline-57 & -22 & 46 & 3.92 & 346 & $\mathrm{~L}$ & Parietal lobe \\
\hline 0 & -76 & 46 & 2.94 & 107 & $L / R$ & Precuneus \\
\hline-54 & -22 & 19 & 5.20 & 497 & $\mathrm{~L}$ & Postcentral gyrus \\
\hline-27 & -1 & 1 & 5.25 & 424 & $\mathrm{~L}$ & Putamen \\
\hline-24 & -67 & -17 & 4.28 & 1188 & $\mathrm{~L}$ & Cerebellum \\
\hline 42 & -43 & 37 & 2.73 & 193 & $\mathrm{R}$ & Parietal lobe \\
\hline 9 & -46 & -11 & 5.33 & 1846 & $\mathrm{R}$ & Cerebellum \\
\hline
\end{tabular}

0.667 , probably new $=0.333$, definitely new $=0)$. We found that a higher trialwise no-go/go network activity ratio predicted poorer subsequent memory, as borne out in a significant negative correlation $\left(\right.$ mean $\left.=-0.59, \mathrm{SEM}=0.28 ; t_{(20)}=2.13, p<0.05\right)$. Thus, as inhibitory demand increased, memory strength for no-go cues decreased. In sum, and in support of the common resource hypothesis, during trials requiring response inhibition, heightened activity (resource demand) in the no-go network, relative to the go network, was predictive of later forgetting of the stimuli encountered on those trials.

Alternatively, another way to approach this question would be to examine whether forgetting of a subset of no-go cues was due to greater mean activity in the go or no-go network alone. This was not the case, as neither the mean activity of the no-go net- work nor the mean activity of the go network alone predicted subsequent memory ( $p>0.05)$ (Table 4$)$. As these tests assume that there is no interaction between the go and no-go networks, these results suggest that inhibition-induced forgetting effect is unlikely to be explained by a single source of variance. Likewise, according to the interactive race model (Boucher et al., 2007), mean activity of the go or no-go network alone would be a poor index of inhibitory demand because it ignores the level of trialby-trial competition between the no-go and go processes. Instead, the level of inhibitory resource demand should directly depend on the relative concurrent activation of the no-go and the go process, as reflected in activity of the no-go and go network respectively (i.e., no-go/go network activity ratio).

As our study focused on examining the resource demands associated with forgetting of no-go cues, we did not have an a priori hypothesis on the association between the no-go/go network activity ratio and subsequent memory of go trials. Exploring this relationship, we found that the activity ratio did not differ between subsequently forgotten and subsequently remembered go cues (go forgotten: mean $=0.41$, go remembered: mean $=$ $\left.0.42, t_{(20)}=0.11, p>0.05\right)$. In accordance with the above finding regarding forgetting of no-go trials, and the present null finding on go trials, an exploratory 2 action cue (no-go, go) $\times 2$ subsequent memory (forgotten, remembered) ANOVA revealed a marginal effect of action cue $\times$ memory interaction $\left(F_{(1,20)}=\right.$ $1.78, p=0.10)$. Additionally, there was a significant main effect of action cue $\left(F_{(1,20)}=8.60, p<0.01, \eta_{P}^{2}=0.30\right)$, which was due to a greater activity ratio for no-go cues compared with go cues (no-go: mean $=0.60$; go: mean $=0.41, t_{(20)}=2.92, p<0.01$, Cohen's $d=0.65)$. The main effect of memory was not significant $\left(F_{(1,20)}=2.15, p>0.5\right)$.

Finally, in addition to linking the no-go/go network activity ratio to subsequent memory, we also assessed the relationship of this metric to ongoing go/no-go task performance. Specifically, given that we assume this ratio to reflect the competition between 
go and no-go processes, we would anticipate a greater no-go/go activity ratio to be associated with delayed responding (slower RT) on go trials. We therefore estimated this relationship between trialwise no-go/go network activity ratio and go RT in each participant, using robust regression (similar to the above analysis involving subsequent memory strength). Although the correlation between the ratio and go RT was positive (mean \pm SEM, $0.27 \pm 0.26$ ), this relationship was not statistically significant at the group level $(p>0.05)$. As a complementary approach of assessing the link between the no-go/go network activity ratio and go RT, we also gauged this relationship at the betweensubject level, by performing a correlation analysis between the mean network activity ratio (in go trials) with the mean go trial RT across participants. As shown in Figure $3 b$, we observed a significant positive correlation (Pearson's $r=0.46, p<0.05$ ), suggesting that greater relative engagement of the no-go versus go network results in slowed responses to go cues, and providing additional support for the sensitivity of this index as a measure of inhibitory resource engagement.

\section{Sapped resources during memory encoding of subsequently forgotten no-go cues}

After demonstrating that subsequently forgotten no-go trials incur exceptionally high inhibitory resource-demand during the go/no-go task, we now turn to examine our second prediction, namely, that these subsequently forgotten no-go trials should also be associated with less activation (indicative of sapped resources) in brain regions associated with memory encoding success.

To identify brain regions that are associated with encoding success of no-go cues, we conducted a conventional DM analysis using the ratings from the surprise recognition memory task. This analysis revealed that encoding success for no-go cues, as expected, was associated with increased activity in visual processing areas (e.g., fusiform gyrus and inferior temporal lobe) and hippocampus, as well as the surrounding medial-temporal lobe (Fig. 4a; Table 5). Additionally, this analysis also revealed bilateral ventrolateral prefrontal cortex (VLPFC, $\sim$ BA45), as well as motor processing areas (supplementary motor area and precentral gyrus) (Fig. $4 a$; Table 5). The reverse contrast examining the subsequent forgetting effect for no-go cues revealed that encoding failure was associated with increased activity in superior frontal gyrus and medial frontal gyrus (Fig. 4b; Table 5). Collectively, these findings converge with many previous studies of episodic memory, which have found encoding success to be associated with increased activity in VLPFC (Henson et al., 1999; Kirchhoff et al., 2000; e.g., Buckner et al., 2001) and encoding failure with activity in superior frontal gyrus (Otten and Rugg, 2001; Wagner and Davachi, 2001).

According to the common resource hypothesis, when more resources are deployed to the response inhibition process, less are left over for memory encoding. Hence, we would expect activity in regions associated with encoding success of no-go cues to exhibit an inverse trial-by-trial relationship with our estimate of inhibitory network resource demand. We therefore performed a correlation analysis between trial-based inhibitory resource demand (i.e., no-go/go network activity ratio) during subsequently

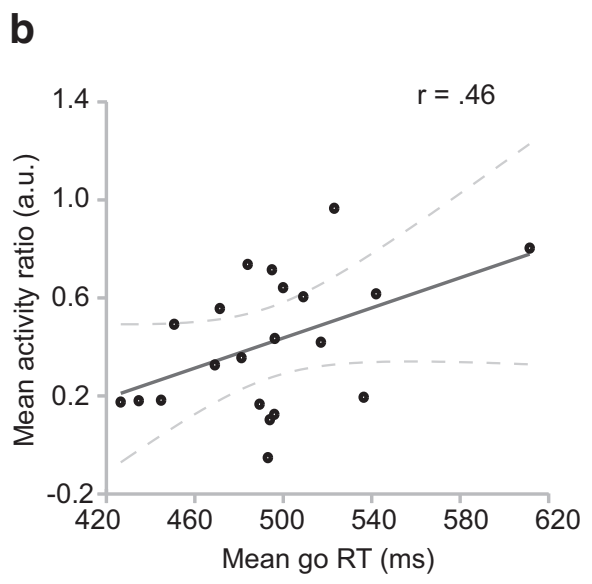

Forgotten

Remembered

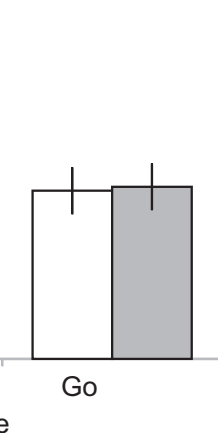

cue

Mean go RT (ms)

for no-go cues

\begin{tabular}{lcl}
\hline & No-go cues & \\
\cline { 2 - 3 } & Forgotten & Remembered \\
\hline Go network & $0.15(0.16)$ & $0.15(0.16)$ \\
No-go network & $-0.04(0.14)$ & $0.07(0.10)$ \\
\hline
\end{tabular}

${ }^{a}$ Data are mean (SEM).

forgotten no-go trials, and activity in voxels within the memory encoding regions (corrected for multiple comparisons within the DM mask), assessing whether these indices were indeed negatively correlated. The manner in which we identified the memory encoding regions (DM analysis) is entirely independent of the computations underlying the trial-by-trial network ratio analysis assessing inhibitory resource demand. Thus, the present correlation analysis was unbiased. In line with our predictions, this analysis revealed a significant negative correlation between inhibitory demand on forgotten no-go trials and activity in a cluster of voxels of the right VLPFC region identified in the DM analysis above (peak MNI $x, y, z=48,44,10 ; t_{(20)}=-2.27, p<0.05$ ) (Fig. $4 c$ ). In this region, mean activity during encoding of subsequently forgotten no-go cues was lower than subsequently remembered no-go cues (Fig. $4 d$ ), as expected from the above analysis. In sum, forgetting of no-go cues, accompanied by higher inhibitory resource-demands, was specifically associated with concurrent decreases in activation in a key frontal brain region involved in the successful encoding of no-go cues (i.e., VLPFC), in line with the idea of neural resources being diverted away from this region during response inhibition to subsequently forgotten no-go cues.

\section{STN does not mediate inhibition-induced forgetting}

Finally, we extracted mean activity from the anatomically defined bilateral STN to test whether the inhibition-induced forgetting effect might be due to a global inhibition process mediated by this structure (Gillies and Willshaw, 1998). If this were the case, we would expect to observe STN activity to be modulated by go/ no-go cues and subsequent memory. However, we did not find any evidence supporting the global inhibition hypothesis. For none of the three levels of ROI mask dilation (to account for variability in anatomy and the normalization procedure across 
a Encoding Success

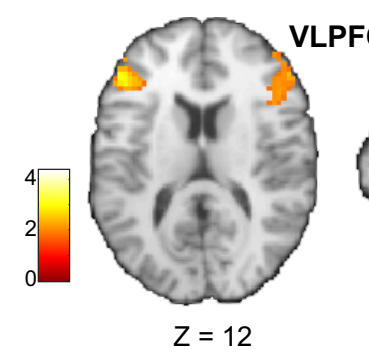

C

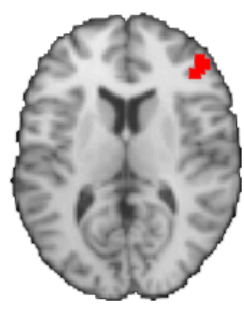

$Z=10$

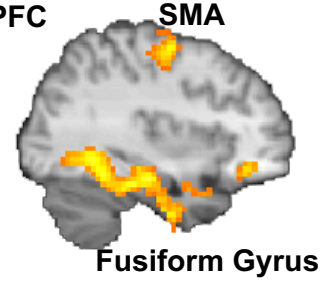

$X=35$

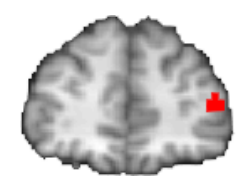

$Y=44$ b Encoding Failure

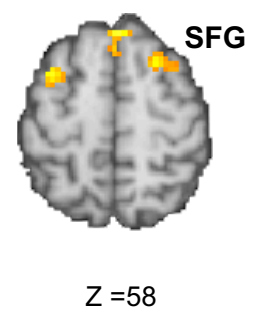

d
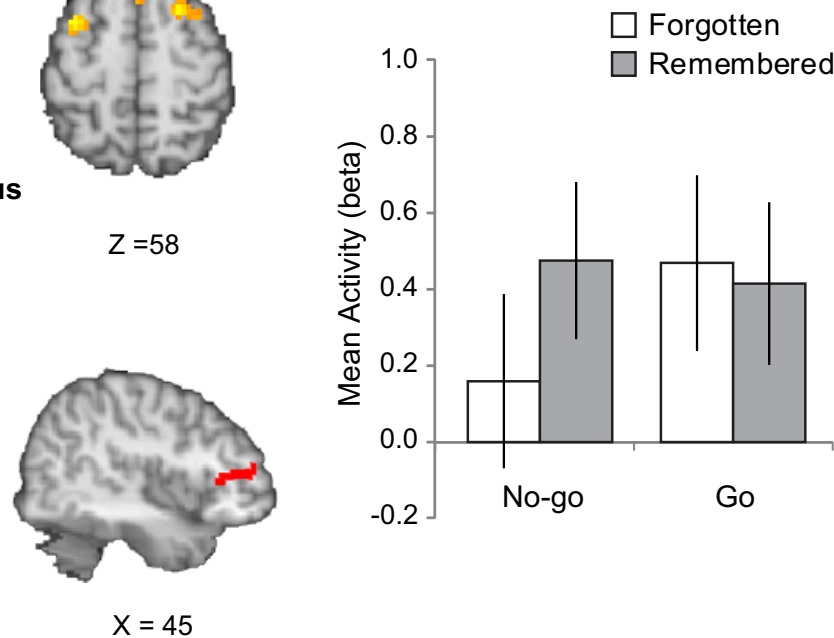

$-0.2$

Figure 4. Memory encoding in relation to inhibitory demands. Brain regions associated with (a) encoding success and $(\boldsymbol{b})$ encoding failure for no-go cues were identified via the conventional difference in memory analysis (whole-brain corrected to $\alpha<0.01$ ). $c$, Of the regions identified in $a$, voxels within the right VLPFC exhibited an inverse trial-by-trial relationship with the index of inhibitory demand (i.e., no-go/go network activity ratio), reflecting sapped resources during the encoding of subsequently forgotten no-go cues. $\boldsymbol{d}$, Bar graph illustrating mean activity $(\beta)$ during go/no-go trials as a function of subsequent memory across all voxels in the cluster shown in c. Image display according to neurological convention (left is left).

Table 5. Activation clusters for subsequent memory effects of no-go cues (wholebrain corrected to $\alpha<0.01$ )

\begin{tabular}{|c|c|c|c|c|c|c|}
\hline \multicolumn{3}{|c|}{ MNI coordinates } & \multirow[b]{2}{*}{ Peak $t$} & \multirow{2}{*}{$\begin{array}{l}\text { No. of } \\
\text { voxels }\end{array}$} & \multirow[b]{2}{*}{ Hemisphere } & \multirow[b]{2}{*}{ Region } \\
\hline$x$ & y & $Z$ & & & & \\
\hline \multicolumn{7}{|c|}{ Remembered $>$ forgotten } \\
\hline 12 & -7 & 76 & 3.74 & 644 & $\mathrm{R}$ & Superior frontal gyrus \\
\hline 36 & -10 & 58 & 3.31 & 255 & $\mathrm{R}$ & Precentral and postcentral gyrus \\
\hline 9 & -10 & 49 & 3.02 & 231 & $\mathrm{R}$ & Supplementary motor area \\
\hline 54 & 44 & 4 & 3.64 & 350 & $\mathrm{R}$ & Inferior frontal gyrus \\
\hline 27 & 35 & -17 & 4.62 & 134 & $\mathrm{R}$ & Middle frontal gyrus \\
\hline 33 & -25 & -20 & 4.57 & 407 & $\mathrm{R}$ & Fusiform gyrus \\
\hline-51 & -19 & 49 & 3.21 & 283 & $\mathrm{~L}$ & Precentral and postcentral gyrus \\
\hline-51 & 35 & 13 & 3.56 & 118 & $\mathrm{~L}$ & Inferior frontal gyrus \\
\hline-57 & -13 & 1 & 3.25 & 97 & $\mathrm{~L}$ & Superior temporal gyrus \\
\hline-37 & -28 & -11 & 3.83 & 169 & $\mathrm{~L}$ & $\begin{array}{l}\text { Hippocampus/parahippocampal } \\
\text { gyrus }\end{array}$ \\
\hline-39 & -22 & -23 & 3.83 & 208 & $\mathrm{~L}$ & Inferior temporal lobe \\
\hline \multicolumn{7}{|c|}{ Forgotten $>$ remembered } \\
\hline 3 & 35 & 55 & 4.29 & 197 & $\mathrm{R}$ & Superior/medial frontal gyrus \\
\hline 24 & 14 & 64 & 2.84 & 102 & $\mathrm{R}$ & Superior frontal gyrus \\
\hline-39 & 11 & 55 & 3.28 & 72 & $\mathrm{~L}$ & Middle frontal gyrus \\
\hline
\end{tabular}

subjects), any significant interactions between go/no-go cues and subsequent memory were observed $(p>0.05)$.

\section{Discussion}

In the current study, we combined a go/no-go task with a surprise recognition memory test to understand the neural mechanisms underlying inhibition-induced forgetting. Behaviorally, no-go cues were remembered more poorly than go cues, replicating recent findings (Chiu and Egner, 2015). We conducted a set of meta-analytic and trial-history analyses to support the notion that no-go trials in our go/no-go task placed demands on response inhibition, despite the fact that we used an equal number of go and no-go trials. Turning to the test of our neural hypotheses, we measured trial-by-trial inhibitory resource-demand by calculating a ratio of no-go to go network activation. We validated this measure as an index of inhibitory resource demand by showing that its magnitude was higher for no-go than for go cues and was associated with slowed go RT across subjects. Importantly, although neither go nor no-go network activity by themselves predicted forgetting of no-go cues, the no-go/go network activity ratio was higher for subsequently forgotten no-go cues than remembered ones, and it furthermore predicted graded memory strength for no-go cues, with a higher ratio during no-go trial performance being associated with poorer subsequent memory for that cue. Collectively, we interpret these finding to indicate that an exceptionally great demand on response inhibition on a subset of no-go trials resulted in a particularly strong relative recruitment of the no-go network, which in turn resulted in unsuccessful memory encoding of stimuli encountered on those trials. It should of course be noted that a high no-go/go network activity ratio can in principle be produced both by relatively high no-go network activity as well as by relatively low go network activity, although in the empirical data, variance in no-go network activity was more prominent (Table 4).

Second, we empirically defined brain regions responsible for successful encoding of no-go cues in a conventional DM analysis, and in a subsequent correlation analysis, we found that activity within one of these regions (the right VLPFC) negatively covaried with the trial-to-trial fluctuation in inhibitory resource-demand, as activity in this region was low when within-trial inhibitory resource-demand was high, and vice versa. Together, this seesaw pattern of resource demand by response inhibition and activity in brain regions recruited for memory encoding supports the hypothesis that the two processes compete for a common resource (or are mutually inhibitory). As more of that resource is expended on response inhibition, less is available to encode the visual stimuli, leading to impoverished memory representation.

We did not observe any evidence to support an alternative "global inhibition" hypothesis because the STN did not exhibit a subsequent memory effect for no-go cues. However, our analysis has two limitations. First, as we increased our chance of including individual participants' STN structure in our group-level anatomical mask by increasing the mask dilation factor, we also increased our 
chance of including noise from non-STN voxels, which could obscure any real effect. Second, our task did not strongly engage STN in the first place. This could be due to the fact that our go/no-go task, unlike the stop signal task, did not require "reactive" stopping (Eagle and Baunez, 2010). Nevertheless, using a stop signal task, we did observe similar inhibition-induced forgetting in our earlier behavioral work (Chiu and Egner, 2015) (Experiment 3), suggesting that response inhibition saps away attentional resources for memory encoding regardless of the specific response inhibition paradigm. Thus, although differential STN activation is clearly not a prerequisite for observing inhibition-induced forgetting, we cannot rule out the possibility that STN might contribute to this effect when observed in a stop-signal task. The latter possibility awaits future investigation. Speaking to the question of stimulus encoding in stop-signal tasks, a recent study documented that the flow of visual stimulus information to anterior brain regions, as gauged by effective connectivity analyses, was relatively suppressed in stop trials (Jahfari et al., 2015). This finding is compatible with the present results, in that both indicate diminished stimulus encoding during response inhibition.

We found converging evidence of subsequent memory being predicted by VLPFC activity. First, we observed greater bilateral VLPFC activity associated with encoding success with the conventional DM analysis. These findings are consistent with many fMRI studies of episodic memory (Kelley et al., 1998; e.g., Henson et al., 1999; Kirchhoff et al., 2000). Second, and most important in the present context, our correlation analysis between activity in these regions and the no-go/go network activity ratio revealed that activity within the right VLPFC was inversely correlated with inhibitory resource-demand on a trial-by-trial basis. This suggests that encoding success is dependent on activity in VLPFC, and its functional role in memory encoding takes a toll when resources are diverted away for implementing response inhibition. It has been proposed that the precise role of the VLPFC in successful encoding lies with selection processes that direct attention toward goal-relevant information (Milner et al., 1985; Miller and Cohen, 2001; Blumenfeld and Ranganath, 2007). Accordingly, VLPFC activation has been found to be negatively correlated with subsequent memory performance if selection processes direct attention away from items that are to be encoded (Otten et al., 2002; Reynolds et al., 2004). The present study corroborates this perspective of VLPFC by directly showing that control demands of response inhibition sap attentional resources that would otherwise be devoted to VLPFC for processes promoting successful encoding.

On the other hand, VLPFC, in particular the right inferior frontal gyrus, has also been associated with the function of response inhibition in go/no-go and stop signal tasks (Aron et al., 2004; Chikazoe, 2010; Cai et al., 2014). However, others have argued that right inferior frontal gyrus is not directly responsible for motoric stopping but rather for the detection of salient or task-relevant cues (Duann et al., 2009; Hampshire et al., 2010), or general context monitoring (Chatham et al., 2012), which is commonly required in response inhibition tasks. To resolve this debate, a recent meta-analysis has suggested that specific forms of cognitive control, including attentional selection, motor inhibition, and action updating, are supported by distinct subregions of the right VLPFC (Levy and Wagner, 2011). Indeed, in our data, no-go trials (contrasted with go trials) activated a more posterior and inferior part of the right VLPFC (BA 45/44) than the region associated with, encoding success (BA 45/47) (compare Figs. $2 a$, $4 a$ ). Therefore, our data also support the proposition that different VLPFC foci contribute to a heterogeneous set of cognitive control processes. Moreover, the present study constitutes neural evidence of capacity sharing based on limitations on attentional selection processes (for review, see Pashler, 1994), which differs from previous localization efforts of a "central bottleneck." The latter has been attributed to the posterior dorsolateral prefrontal cortex (BA9), based on limitations in the ability to select two responses under dual-task situations (Dux et al., 2006). Given that there were no dual-task or response requirements in our study, the present results complement those results and document a resource-based performance limitation that is based on competition between attentional prioritization of action control versus memory encoding.

The finding of inhibition-induced forgetting adds to an emerging literature using incidental memory as a window onto the architecture of cognitive control processes. Notably, inhibition-induced forgetting stands in contrast to the mnemonic consequences of another core cognitive control process, namely, "conflict-control," the detection and resolution of conflict between incompatible responses or stimulus representations (Botvinick et al., 2001). Conflict-control has been shown to enhance subsequent memory for task-relevant stimuli, as incidental memory tends to be higher for task-relevant targets presented along with incongruent distractors than for targets presented with congruent distractors (Krebs et al., 2015; Rosner et al., 2015). This finding is in line with the proposal that conflict in information processing triggers the reinforcement of top-down attention toward task-relevant stimuli (Botvinick et al., 2001; Egner and Hirsch, 2005), resulting in superior subsequent memory for those stimuli. fMRI data have supported this view, showing that superior conflict-enhanced target memory is predicted by conflict-triggered activity in dorsolateral prefrontal and parietal cortices (Krebs et al., 2015). In combination with the present results, these data clearly support the notion that the control processes subserved by dorsolateral prefrontal areas during conflict resolution differ in nature from the processes underpinned by the VLPFC regions that form part of the no-go network. Whereas conflictcontrol summons attention toward task-relevant stimuli, response inhibition diverts attention away from those stimuli, presumably toward action control processes.

In conclusion, by examining the mnemonic consequences of inhibitory control, our study supports a common resource hypothesis whereby the exertion of response inhibition saps attentional resources away from stimulus encoding, causing later forgetting of (some) stop cues. This competition for common resources is reflected by a seesaw pattern between neural inhibitory resource-demand and activity in brain regions supporting memory encoding processes. As more central resources are demanded by inhibitory control, less can be devoted to memory encoding processes served by the VLPFC.

\section{References}

Aron AR (2007) The neural basis of inhibition in cognitive control. Neuroscientist 13:214-228. CrossRef Medline

Aron AR, Robbins TW, Poldrack RA (2004) Inhibition and the right inferior frontal cortex. Trends Cogn Sci 8:170-777. CrossRef Medline

Aron AR, Behrens TE, Smith S, Frank MJ, Poldrack RA (2007) Triangulating a cognitive control network using diffusion-weighted magnetic resonance imaging (MRI) and functional MRI. J Neurosci 27:3743-3752. CrossRef Medline

Blumenfeld RS, Ranganath C (2007) Prefrontal cortex and long-term memory encoding: an integrative review of findings from neuropsychology and neuroimaging. Neuroscientist 13:280-291. CrossRef Medline

Botvinick MM, Braver TS, Barch DM, Carter CS, Cohen JD (2001) Conflict monitoring and cognitive control. Psychol Rev 108:624-652. CrossRef Medline

Boucher L, Palmeri TJ, Logan GD, Schall JD (2007) Inhibitory control in 
mind and brain: an interactive race model of countermanding saccades. Psychol Rev 114:376-397. CrossRef Medline

Buckner RL, Wheeler ME, Sheridan MA (2001) Encoding processes during retrieval tasks. J Cogn Neurosci 13:406-415. CrossRef Medline

Cai W, Ryali S, Chen T, Li CS, Menon V (2014) Dissociable roles of right inferior frontal cortex and anterior insula in inhibitory control: evidence from intrinsic and task-related functional parcellation, connectivity, and response profile analyses across multiple datasets. J Neurosci 34:1465214667. CrossRef Medline

Chatham CH, Claus ED, Kim A, Curran T, Banich MT, Munakata Y (2012) Cognitive control reflects context monitoring, not motoric stopping, in response inhibition. PLoS One 7:e31546. CrossRef Medline

Chikazoe J (2010) Localizing performance of go/no-go tasks to prefrontal cortical subregions. Curr Opin Psychiatry 23:267-272. CrossRef Medline

Chiu YC, Egner T (2015) Inhibition-induced forgetting: when more control leads to less memory. Psychol Sci 26:27-38. CrossRef Medline

de Wit H (2009) Impulsivity as a determinant and consequence of drug use: a review of underlying processes. Addict Biol 14:22-31. CrossRef Medline

Duann JR, Ide JS, Luo X, Li CS (2009) Functional connectivity delineates distinct roles of the inferior frontal cortex and presupplementary motor area in stop signal inhibition. J Neurosci 29:10171-10179. CrossRef Medline

Durston S, Thomas KM, Worden MS, Yang Y, Casey BJ (2002) The effect of preceding context on inhibition: an event-related fMRI study. Neuroimage 16:449-453. CrossRef Medline

Dux PE, Ivanoff J, Asplund CL, Marois R (2006) Isolation of a central bottleneck of information processing with time-resolved fMRI. Neuron 52: 1109-1120. CrossRef Medline

Eagle DM, Baunez C (2010) Is there an inhibitory-response-control system in the rat? Evidence from anatomical and pharmacological studies of behavioral inhibition. Neurosci Biobehav Rev 34:50-72. CrossRef Medline

Egner T, Hirsch J (2005) Cognitive control mechanisms resolve conflict through cortical amplification of task-relevant information. Nat Neurosci 8:1784-1790. CrossRef Medline

Endl W, Walla P, Lindinger G, Lalouschek W, Barth FG, Deecke L, Lang W (1998) Early cortical activation indicates preparation for retrieval of memory for faces: an event-related potential study. Neurosci Lett 240: 58-60. CrossRef Medline

Friston KJ, Holmes AP, Worsley KJ, Poline JP, Frith CD, Frackowiak RSJ (1994) Statistical parametric maps in functional imaging: a general linear approach. Hum Brain Mapp 2:189-210. CrossRef

Garavan H, Ross TJ, Stein EA (1999) Right hemispheric dominance of inhibitory control: an event-related functional MRI study. Proc Natl Acad Sci U S A 96:8301-8306. CrossRef Medline

Gillies AJ, Willshaw DJ (1998) A massively connected subthalamic nucleus leads to the generation of widespread pulses. Proc Biol Sci 265:2101-2109. CrossRef Medline

Hampshire A, Chamberlain SR, Monti MM, Duncan J, Owen AM (2010) The role of the right inferior frontal gyrus: inhibition and attentional control. Neuroimage 50:1313-1319. CrossRef Medline

Henson RN, Rugg MD, Shallice T, Josephs O, Dolan RJ (1999) Recollection and familiarity in recognition memory: an event-related functional magnetic resonance imaging study. J Neurosci 19:3962-3972. Medline

Horn NR, Dolan M, Elliott R, Deakin JF, Woodruff PW (2003) Response inhibition and impulsivity: an fMRI study. Neuropsychologia 41:19591966. CrossRef Medline

Jahfari S, Waldorp L, Ridderinkhof KR, Scholte HS (2015) Visual information shapes the dynamics of cortico-basal ganglia pathways during response selection and inhibition. J Cogn Neurosci 27:1344-1359. CrossRef Medline

Jurado MB, Rosselli M (2007) The elusive nature of executive functions: a review of our current understanding. Neuropsychol Rev 17:213-233. CrossRef Medline

Kanade T, Cohn JF (2000) Comprehensive database for facial expression analysis. In: Proceedings of the Fourth International Conference on Automatic Face and Gesture Recognition (FG'00), pp 46-53. Grenoble, France: IEEE.

Kelley WM, Miezin FM, McDermott KB, Buckner RL, Raichle ME, Cohen NJ, Ollinger JM, Akbudak E, Conturo TE, Snyder AZ, Petersen SE (1998) Hemispheric specialization in human dorsal frontal cortex and medial temporal lobe for verbal and nonverbal memory encoding. Neuron 20: 927-936. CrossRef Medline
Kirchhoff BA, Wagner AD, Maril A, Stern CE (2000) Prefrontal-temporal circuitry for episodic encoding and subsequent memory. J Neurosci 20: 6173-6180. Medline

Konishi S, Nakajima K, Uchida I, Kikyo H, Kameyama M, Miyashita Y (1999) Common inhibitory mechanism in human inferior prefrontal cortex revealed by event-related functional MRI. Brain 122:981-991. CrossRef Medline

Krebs RM, Boehler CN, De Belder M, Egner T (2015) Neural conflictcontrol mechanisms improve memory for target stimuli. Cereb Cortex 25:833-843. CrossRef Medline

Levy BJ, Wagner AD (2011) Cognitive control and right ventrolateral prefrontal cortex: reflexive reorienting, motor inhibition, and action updating. Ann N Y Acad Sci 1224:40-62. CrossRef Medline

Liddle PF, Kiehl KA, Smith AM (2001) Event-related fMRI study of response inhibition. Hum Brain Mapp 12:100-109. CrossRef Medline

Logan GD, Cowan WB (1984) On the ability to inhibit thought and action: a theory of an act of control. Psychol Rev 91:295-327. CrossRef Medline

Maldjian JA, Laurienti PJ, Kraft RA, Burdette JH (2003) An automated method for neuroanatomic and cytoarchitectonic atlas-based interrogation of fMRI data sets. NeuroImage 19:1233-1239. Medline

Mansfield EL, Karayanidis F, Jamadar S, Heathcote A, Forstmann BU (2011) Adjustments of response threshold during task switching: a model-based functional magnetic resonance imaging study. J Neurosci 31:1468814692. CrossRef Medline

Miller EK, Cohen JD (2001) An integrative theory of prefrontal cortex function. Annu Rev Neurosci 24:167-202. CrossRef Medline

Milner B, Petrides M, Smith ML (1985) Frontal lobes and the temporal organization of memory. Hum Neurobiol 4:137-142. Medline

Minear M, Park DC (2004) A lifespan database of adult facial stimuli. Behav Res Methods Instrum Comput 36:630 -633. CrossRef Medline

Mink JW (1996) The basal ganglia: focused selection and inhibition of competing motor programs. Prog Neurobiol 50:381-425. CrossRef Medline

Miyake A, Friedman NP (2012) The nature and organization of individual differences in executive functions: four general conclusions. Curr Direct Psychol Sci 21:8-14. CrossRef Medline

Mumford JA, Turner BO, Ashby FG, Poldrack RA (2012) Neuroimage deconvolving BOLD activation in event-related designs for multivoxel pattern classification analyses. Neuroimage 59:2636-2643. CrossRef Medline

Otten LJ, Rugg MD (2001) When more means less: neural activity related to unsuccessful memory encoding. Curr Biol 11:1528-1530. CrossRef Medline

Otten LJ, Henson RN, Rugg MD (2002) State-related and item-related neural correlates of successful memory encoding. Nat Neurosci 5:1339-1344. CrossRef Medline

Paller KA, Wagner AD (2002) Observing the transformation of experience into memory. Trends Cogn Sci 6:93-102. CrossRef Medline

Pashler H (1994) Dual-task interference in simple tasks: data and theory. Psychol Bull 116:220-244. Medline

Reynolds JR, Donaldson DI, Wagner AD, Braver TS (2004) Item- and tasklevel processes in the left inferior prefrontal cortex: positive and negative correlates of encoding. Neuroimage 21:1472-1483. CrossRef Medline

Rissman J, Gazzaley A, D’Esposito M (2004) Measuring functional connectivity during distinct stages of a cognitive task. Neuroimage 23:752-763. CrossRef Medline

Rosner TM, D'Angelo MC, MacLellan E, Milliken B (2015) Selective attention and recognition: effects of congruency on episodic learning. Psychol Res 79:411-424. CrossRef Medline

Rubia K, Smith AB, Brammer MJ, Taylor E (2003) Right inferior prefrontal cortex mediates response inhibition while mesial prefrontal cortex is responsible for error detection. Neuroimage 20:351-358. CrossRef Medline

Thomas SJ, Gonsalvez CJ, Johnstone SJ (2009) Sequence effects in the Go/ NoGo task: inhibition and facilitation. Int J Psychophysiol 74:209-219. CrossRef Medline

Verbruggen F, Logan GD (2008) Automatic and controlled response inhibition: associative learning in the go/no-go and stop-signal paradigms. J Exp Psychol 137:649-672. CrossRef Medline

Wagner AD, Davachi L (2001) Cognitive neuroscience: forgetting of things past. Curr Biol 11:R964-R967. CrossRef Medline

Yarkoni T, Poldrack RA, Nichols TE, Van Essen DC, Wager TD (2011) Large-scale automated synthesis of human functional neuroimaging data. Nat Methods 8:665-670. CrossRef Medline 